\title{
A Wholly Analytical Method for the Simulation of an Electromagnetic Acoustic Transducer Array
}

DOI:

10.3233/JAE-150137

\section{Document Version}

Accepted author manuscript

Link to publication record in Manchester Research Explorer

\section{Citation for published version (APA):}

Xie, Y., Yin , L., Rodriguez Gutierrez, S., Yang, T., Liu, Z., \& Yin, W. (2016). A Wholly Analytical Method for the Simulation of an Electromagnetic Acoustic Transducer Array. International Journal of Applied Electromagnetics and Mechanics (IOS Press), 51(4), 375-389. https://doi.org/10.3233/JAE-150137

\section{Published in:}

International Journal of Applied Electromagnetics and Mechanics (IOS Press)

\section{Citing this paper}

Please note that where the full-text provided on Manchester Research Explorer is the Author Accepted Manuscript or Proof version this may differ from the final Published version. If citing, it is advised that you check and use the publisher's definitive version.

\section{General rights}

Copyright and moral rights for the publications made accessible in the Research Explorer are retained by the authors and/or other copyright owners and it is a condition of accessing publications that users recognise and abide by the legal requirements associated with these rights.

\section{Takedown policy}

If you believe that this document breaches copyright please refer to the University of Manchester's Takedown Procedures [http://man.ac.uk/04Y6Bo] or contact uml.scholarlycommunications@manchester.ac.uk providing relevant details, so we can investigate your claim.

\section{OPEN ACCESS}




\title{
A Wholly Analytical Method for the Simulation of an Electromagnetic
}

\section{Acoustic Transducer Array}

\author{
Yuedong XIE ${ }^{1}$, Liyuan YIN², Sergio RODRIGUEZ G ${ }^{1}$, Tiemei YANG ${ }^{3}$, Zenghua LIU $^{4}$, \\ Wuliang $\mathrm{YIN}^{1 *}$ \\ ${ }^{1}$ School of Electrical and Electronic Engineering, University of Manchester, Manchester, United Kingdom, \\ M13 9PL \\ ${ }^{2}$ Kunming University of Science and Technology, Kunming, China. \\ ${ }^{3}$ Taiyuan University of Science and Technology, Shanxi, 030024, China \\ ${ }^{4}$ College of Mechanical Engineering and Applied Electronics Technology, Beijing University of Technology, \\ Beijing, 100124, China. \\ * Corresponding Author: E-mail: wuliang.yin@ manchester.ac.uk; Fax number: +44 (0) 161306 4644; \\ Telephone number: +44 (0) 161 -3062885;
}

\begin{abstract}
This paper presents a wholly analytical method which combines electromagnetic and ultrasonic simulations for the study of an Electromagnetic Acoustic Transducer Array (EMAT). Analytical solutions to the eddy current problem for the meander coil used in an EMAT are adapted from the classic Deeds and Dodd solution, which was originally intended for circular coils. The analytical solution resulting from this novel adaptation exploits the large radius extrapolation and shows several advantages over the finite element method (FEM), especially in the higher frequency regime. The calculated Lorentz force density from the EM solver is then coupled to the ultrasonic simulations, which exploit an analytical solution for generating the distribution of surface waves. Beam features are studied, which can produce performance parameters for an EMAT array, facilitating the optimum design of such sensors. This total analytical approach to an EMAT simulation has not been reported previously to our knowledge.
\end{abstract}


Keywords: Eddy current calculation, Modelling and Simulation, Electromagnetic acoustic transducer (EMAT), Analytical solutions, Finite element method (FEM)

\section{Introduction}

There are a variety of non-destructive testing (NDT) techniques employed in industries, such as magnetic particle inspection (MPI), electromagnetic methods (EM), eddy current methods, and ultrasonic methods [1-7]. Due to its advantages of having good penetration depth and mechanical flexibility, the piezoelectric ultrasonic method is widely used for thickness measurement, flaw evaluation and material characterization [8-13]. The transducer frequently used is made of piezoelectric ceramics or crystals [12-15]. However, one primary disadvantage of the piezoelectric ultrasonic testing is the need to have good sonic contact with the test piece, typically by means of a couplant for acoustic impedance matching [16].

Electromagnetic acoustic transducers (EMATs) are becoming increasingly popular due to their non-contact nature [17-21]. An EMAT sensor typically consists of a permanent magnet providing a large static magnetic field and a coil carrying an alternating current which is placed next to the test piece [22-24]. There are two EMAT interactions which can produce ultrasound: magnetostriction for magnetic materials and the Lorentz force mechanism for conducting metallic materials $[17,23,25]$. Because an EMAT generates ultrasonic waves directly into the testing piece instead of coupling through the transducer, an EMAT has advantages in applications where surface contact is not possible or desirable [26, 27]. Another attractive feature of EMAT is a variety of wave modes can be produced based on different combinations of coils and magnets $[17,28]$. In this paper, only EMAT based on Lorentz force mechanism to generate surface waves is discussed.

Considerable works were reported on EMAT modelling [29-33]. All of these papers divide EMAT modelling into two parts, electromagnetic simulation to obtain Lorentz force density 
and ultrasonic simulation to model the ultrasonic wave propagation due to Lorentz force. Electromagnetic simulation can be achieved by the finite element method (FEM) and the analytical method [34-36]; ultrasonic simulation can be performed with the finite element method (FEM), finite-difference time-domain (FDTD), and the analytical method [17, 37, 38]. Some papers combine the finite element method (FEM) and the analytical method to model EMAT; that is, the finite element method (FEM) is used to carry out electromagnetic simulation and the analytical method is to achieve ultrasonic simulation [33, 37, 39, 40]. Some papers model EMAT arrays with the finite element method (FEM) for both electromagnetic and ultrasonic simulations, that is, the implicit finite element software COMSOL for the electromagnetic simulation and the explicit finite element software Abaqus for the ultrasonic simulation $[17,25]$. The summary of the state of the art methods used for EMAT modelling is shown in Table 1; the method combining finite element method (FEM) and finite-difference time-domain (FDTD) proposed by authors has been published in [41], the method combining the analytical method and the finite-difference time-domain (FDTD) proposed by authors has been published in [42], and the method wholly using analytical solutions to model EMAT arrays have not been studied before.

Consequently, this paper proposes a new method using analytical solutions, both for the electromagnetic simulation and for the ultrasonic simulation, to model EMAT arrays. On one hand, the EM analytical approach is used to calculate Lorentz force density for a given coil and a particular DC biased magnet configuration, which then can be fed through to ultrasonic simulations. On the other hand, an analytical method describing the ultrasonic wave propagation due to the EM Lorentz force density acting upon the metallic sample is used for the ultrasonic simulation for an EMAT.

\section{Analytical method for eddy current calculation}




\subsection{The governing Equations}

C. V. Dodd and W. E. Deeds proposed analytical solutions to the circular coil over an layered conductor in [35]. The geometry used for studying analytical solutions is shown in Figure 1, where a circular coil is placed above the test piece made of aluminium. $r_{1}$ and $r_{2}$ are the inner and outer radius of the circular coil, $h$ is the height of the coil, $l$ is the lift-off distance, $R$ and $H$ is the length and height of aluminium respectively.

The governing equations for induced eddy current calculation are,

$$
\begin{array}{r}
\nabla^{2} \mathbf{A}=-\mu \mathbf{I}+\mu \sigma \frac{\partial \mathbf{A}}{\partial t}+\mu \epsilon \frac{\partial^{2} \mathbf{A}}{\partial t^{2}}+\mu \nabla\left(\frac{1}{\mu}\right) \times(\nabla \times \mathbf{A}) \\
\mathbf{E}=-j \omega \mathbf{A} \ldots \ldots \ldots \ldots \ldots \ldots \ldots \ldots \ldots \ldots \ldots \ldots \ldots \ldots \ldots
\end{array}
$$

where $\mathbf{A}$ is the vector potential, $\mu, \sigma$ and $\epsilon$ is the permeability, conductivity and permittivity of the material respectively, I is the applied current density, $\omega$ is the angular frequency of the applied alternating current, $\mathbf{E}$ is the electric field, and $\mathbf{J}$ is the induced eddy current.

From Equations (1), (2) and (3), for eddy current calculation, the main thing is to calculate the vector potential. C. V. Dodd and W. E. Deeds provided the final analytical solutions to the vector potential calculation; after simplifying, the vector potential within the conductor can be described as following [35],

$$
\begin{aligned}
& \mathbf{A}(r, z)=\mathbf{M} \int_{0}^{\infty} \frac{1}{a^{2}}\left(\int_{a r_{1}}^{a r_{2}} x J_{1}(x) d x\right) J_{1}(a r)\left(e^{-a l}-e^{-a(l+h)}\right) \frac{e^{a_{1} z}}{a+a_{1}} d a \ldots \\
& \mathbf{M}=\mu_{0} N \mathbf{I} \\
& a_{1}=\sqrt{a^{2}+j \omega \mu_{1} \sigma}
\end{aligned}
$$


where $N$ is the turns of the coil, $a$ and $x$ are the integration variables, $J_{1}(a r)$ and $J_{1}(x)$ are the Bessel functions of first kind. $\mu_{0}$ and $\mu_{1}$ are the permeability of air and conductor respectively. $\sigma$ is the conductivity of the conductor.

We build a model to study the analytical solutions to the vector potential problem; the parameters used are listed in Table 2. By calculating with Matlab, the magnitude distribution of the vector potential A along the surface of aluminium $(z=0)$ is shown in Figure 2, where the vector potential is mainly concentrated under the circular coil; the unit of the vector potential is teslaxmeter. The red point in Figure 2 means the maximum vector potential, which shows the distribution of the vector potential is not symmetrical with the radius $r=5 \mathrm{~mm}$ because the wire of the circular coil is not straight.

\subsection{Analytical method for meander coil}

In this work, the coil used in an EMAT is a meander coil, so the analytical solutions to a straight wire are needed. Based on the analytical solutions proposed by Dodd and Deeds, we proposed an assumption, that is, when the radius of the circular coil is very large, the bent wire of the circular coil can be approximated to a straight wire, and the distribution of the vector potential would be symmetrical. To verify this assumption, we build a model with the same parameters used in Table 2, except that the mean radius of the circular coil is $10.05 \mathrm{~m}$, and the range of the aluminium sample is from $10 \mathrm{~m}$ to $10.1 \mathrm{~m}$. The magnitude distribution of the vector potential A along the surface $(z=0)$ from $10 \mathrm{~m}$ to $10.1 \mathrm{~m}$ is shown in Figure 3. From this figure, the magnitude distribution is symmetrical with $r=10.05 \mathrm{~m}$, where the wire of the coil is located. This verifies the assumption, that is, when the radius of the spiral coil is very large, the bend wire can be approximated to a straight wire and the solution of Deeds and Dodd can be adapted for this application. 


\subsubsection{Comparison with FEM at $1 \mathrm{kHz}$}

For a straight wire solution, in order to compare its analytical solution to the finite element method (FEM), Maxwell Ansoft is used to construct a model with the same parameters used in 2.2; the FEM model is built with a straight wire located above the test piece. The distributions of the vector potential with the numerical method and the analytical method are shown in Figure 4(a) and Figure 4(b) respectively. In Figure 4, both the finite element method (FEM) and the analytical method show that the maximum vector potential occurs at the surface of aluminium and decreases along the depth. The magnitude range of the vector potential based on the analytical method is consistent with that of the finite element method (FEM), which is from 0 to $2.5 \times 10^{-7}$ tesla $\times$ meter.

On the surface of aluminium $(z=0)$, the distribution of the vector potential is shown in Figure 5, where the magnitude, the real part and the imaginary part of the vector potential distribution are shown in Figure 5(a), (b) and (c) respectively. In Figure 5(a), for magnitude distribution, the analytical method and the finite element method are consistent. However, for the real part and the imaginary part of the vector potential, as shown in Figure 5(b) and Figure 5(c), the analytical method shows a more accurate result than FEM because the vector potential based on FEM is not approaching zero when A is away from the wire.

\subsubsection{Comparison with FEM at $1 \mathrm{MHz}$}

In this part, the analytical solutions at a high operating frequency are studied. This is because EMAT normally operates at high frequencies and the eddy current is typically limited near the surface. The model used is the same with that in 2.2.1, except that the frequency used is $1 \mathrm{MHz}$.

Along the surface of aluminium $(z=0)$, the distribution of the vector potential based on the analytical method and the finite element method (FEM) is shown in Figure 6. For the 
magnitude of the vector potential, the curve obtained by the analytical method is smoother than that of FEM; this is due to the inevitable error of numerical approximation. This observation is further verified by the plot of the real and imaginary part distribution, as shown in Figure 6(b) and Figure 6(c) respectively.

\subsubsection{Comparison with FEM at a depth larger than the skin depth}

In addition, the distributions of the vector potential at a depth larger than the skin depth are analysed in this part. Skin depth can be calculated by Equation (7).

$$
d_{s d}=\sqrt{\frac{2}{\omega \sigma \mu_{0} \mu_{1}}}
$$

where $\omega$ is the angular frequency, $\sigma$ is the conductivity of the conductor, $\mu_{0}$ is the permeability of free space and $\mu_{1}$ is the conductor's relative permeability. The frequency used is $1 \mathrm{kHz}$, and the calculated skin depth is $2.58 \mathrm{~mm}$. Any depth out of the skin depth can be chosen to study the vector potential distribution at a depth larger than the skin depth, here a depth of $3 \mathrm{~mm}$ is chosen; the distribution of the vector potential at $\mathrm{z}=-3 \mathrm{~mm}$ is shown in Figure 7 . From these plots, the curve of the vector potential distribution based on the analytical method is smoother than that of FEM. Even when the mesh of FEM is very fine, the results based on FEM is not as good as those based on the analytical method.

With the comparisons of the results at $1 \mathrm{kHz}$ and $1 \mathrm{MHz}$, as shown in Figure 5 and Figure 6 respectively, FEM becomes less reliable at a high frequency, even if the mesh is very fine; that is because of the numerical nature of the FEM: numerical approximation due to finite mesh density and element interpolation are inevitable. However, the analytical method shows good results, no matter at a low frequency or a high frequency. In all, the analytical method provides several advantages over FEM, including maintaining high accuracy for the high frequency regime, and showing good results at a depth larger than the skin depth. 


\section{EMAT modelling}

An EMAT sensor consists basically of a coil carrying an alternating current, a permanent magnet providing a large static magnetic field, and the test piece, as shown in Figure 8 . The coil induces eddy currents $\mathbf{J}$ in the surface layers of the testing material, and the interaction between the static magnetic field $\mathbf{B}$ and eddy currents $\mathbf{J}$ produces a Lorentz force density $\mathbf{F}$ based on Equation (8), which in turn generates ultrasound waves propagating within the testing sample.

$$
\mathbf{F}=\mathbf{J} \times \mathbf{B}
$$

In this work, the EMAT modelling consists of two simulations, electromagnetic simulation and ultrasonic simulation. Electromagnetic simulation is used to obtain Lorentz force density, which is the link between electromagnetic simulation and ultrasonic simulation. Both electromagnetic simulation and ultrasonic simulations are achieved by the analytical method.

The testing sample used is a stainless steel plate with a dimension of $1000 \times 1000 \times 3 \mathrm{~mm}^{3}$, and the permanent magnet used is $\mathrm{NdFeB} 35$, whose size is $80 \times 80 \times 30 \mathrm{~mm}^{3}$. The meander coil carries an alternating current with the peak of $50 \mathrm{~A}$, the lift-off is $1 \mathrm{~mm}$, the operation frequency is $500 \mathrm{kHz}$, and the skin depth calculated is $0.679 \mathrm{~mm}$. The Rayleigh wave velocity is 3.033 $\mathrm{mm} / \mu \mathrm{s}$ in the stainless steel plate used, so the wavelength of Rayleigh waves is $6.066 \mathrm{~mm}$; the centre-to-centre distance between two adjacent lines of the meander coil is equalling to one half of the Rayleigh wavelength, which is $3.033 \mathrm{~mm}$, to form the constructive interference.

\subsection{Electromagnetic simulation}

In electromagnetic simulation, the meander coil used has a dimension of $20 \times 34.163 \times 0.036$ $\mathrm{mm}^{3}$, which is very small compared to the size of the stainless steel plate. In order to improve modelling time, only the area $\left(100 \times 100 \times 3 \mathrm{~mm}^{3}\right)$ where the meander coil has a major effect on 
is picked to study the Lorentz force distribution. The subsequent electromagnetic simulations are carried out in 2-D on the $y-z$ cross-section of the stainless steel plate.

As mentioned before, the distribution of the vector potential under a straight wire can be obtained by analytical solutions. For a meander coil, the total vector potential is the sum of the vector potential caused by each wire segment; the distribution of the vector potential on $y-z$ section of the stainless steel plate is shown in Figure 9, where fields between two adjacent wires are opposite due to opposite directions of the alternating currents. In addition, the values of the vector potential under the outmost wires are the largest, because the outmost wires are only affected by the fields on one side.

Along the surface of the stainless steel plate, Lorentz force density distribution is shown in Figure 10, which confirms the observation that the amplitude along the outmost wire is largest. Because there are six pairs of adjacent wires with different current directions, the curve has 6 crests and 6 troughs. It can be seen that the Lorentz force density on the outmost lines is larger than that on the inner lines; that's because both the maximum magnetic field and the maximum eddy current occur at the places corresponding to the edges of the EMAT sensor.

\subsection{Ultrasonic simulation}

Analytical solutions to the propagation of surface waves are given by [43], which defines the surface waves' displacement due to an applied force along $y$ axis. As shown in Figure 11, a surface point force along $y$ axis is applied on point A, which generates ultrasound waves. At an arbitrary point B, the displacement of surface waves is obtained by Equations (9) and (10).

$$
\begin{aligned}
& \boldsymbol{d}_{\boldsymbol{r}}=p * \mathbf{F} * \frac{\beta_{t} \beta_{r} \gamma_{1}}{A^{\prime}\left(\beta_{r}\right) \sqrt{\beta_{r} r}} e^{\left[j\left(\omega t-\beta_{r} r+\frac{\pi}{4}\right)\right]} \cos \theta \\
& \boldsymbol{d}_{\boldsymbol{t}}=p * \mathbf{F} * \frac{\beta_{t} \beta_{r} \gamma_{1}}{A^{\prime}\left(\beta_{r}\right) \sqrt[3]{\beta_{r} r}} e^{\left[j\left(\omega t-\beta_{r} r-\frac{\pi}{4}\right)\right]} \sin \theta .
\end{aligned}
$$


where $\boldsymbol{d}_{\boldsymbol{r}}$ and $\boldsymbol{d}_{\boldsymbol{t}}$ is the radial displacement and tangential displacement respectively; $p=$ $j \beta_{t} /(\sqrt{2 \pi} \mu), \mu=\rho v_{t}^{2}$, where $\rho$ is the density of the conductor; $\mathbf{F}$ is the applied point force; $\beta_{t}=\frac{\omega}{v_{t}}, \beta_{r}=\frac{\omega}{v_{r}}, \beta_{l}=\frac{\omega}{v_{l}}$, where $v_{l}, v_{t}$ and $v_{r}$ are the velocities of the longitudinal waves, transverse waves and surface waves respectively, and $\omega$ is the angular frequency; $\gamma_{1}=$ $\sqrt{\beta_{r}{ }^{2}-\beta_{t}{ }^{2}} ; A^{\prime}\left(\beta_{r}\right)=\frac{\partial\left[\left(2 \beta_{r}{ }^{2}-\beta_{t}{ }^{2}\right)^{2}-4 \beta_{r}{ }^{2} \sqrt{\left(\beta_{r}{ }^{2}-\beta_{t}{ }^{2}\right)\left(\beta_{r}{ }^{2}-\beta_{l}{ }^{2}\right)}\right]}{\partial\left(\beta_{r}\right)} ; r$ is the distance between point A and $\mathrm{B}, \theta$ is the angle between $r$ and $\mathbf{F}$. In addition, if there are multiple point forces on the surface, the total displacement at an arbitrary point B is the superposition of the displacement caused by each point force.

In this work, Lorentz force density obtained from the electromagnetic simulation is imported to the ultrasonic model to generate surface waves. The ultrasonic simulations based on analytical solutions are carried out in 2D on the $x-y$ cross-section of the stainless steel plate; as shown in Figure 12, the 12 alternating Lorentz force densities are added to the ultrasonic model along the meander coil. In the ultrasonic modelling, because the outmost lines of the meander coil are longer than the inner lines of the meander coil, the outmost lines should have more points than the inner lines; in this work, a total number of 74 points with 6 points along the inner lines and 7 points along the outmost lines are used (as shown in Figure 12). The original $x$-y cross-section is $1000 * 1000 \mathrm{~mm}^{2}$; in order to save modelling geometry and improve modelling time, only the area $\left(150 * 1000 \mathrm{~mm}^{2}\right)$ where surface waves mainly propagating is modelled.

The amplitude distribution of surface waves on the stainless steel plate is shown in Figure 13 (a), where surface waves are mainly propagating along $y$ axis. The amplitude distribution of surface waves can be used to study beam features, including beam directivity and field distribution along the steering angle. Beam directivity is, at a radial length from the centre of the EMAT sensor, the displacement distribution along the red circle in Figure 13(b); we define 
the angle of the maximum displacement as steering angle. Field distribution along the steering angle is the displacement distribution along the steering angle, as shown in the blue line in Figure 13(b). Figure 14 shows the results of the beam directivity at a radial distance of $35 \mathrm{~mm}$ and displacement distribution along the steering angle. From the plot of beam directivity (Figure 14(a)), surface waves are mainly propagating along $0^{\circ}$ and $180^{\circ}$, which are named steering angles in this work; the unit of the displacement amplitude in the radial direction is millimetre. At the steering angles, $0^{\circ}$ and $180^{\circ}$, the displacement distribution is shown in Figure 14(b); at the centre of the sensor, the interference of surface waves generated by each wire are destructive, so the amplitude is very small at the centre of the sensor $(y=500 \mathrm{~mm})$ as shown in Figure 14(b). With the radial distance from the centre of the sensor increasing, the amplitude increases as well because surface waves are in the process of constructive interference. When the constructive interference between all wires is finished totally, the amplitude arrives to peak. After that, the amplitude decreases due to the attenuation of surface waves.

\section{Conclusion}

A method using the analytical method for both electromagnetic and ultrasonic simulations to model an EMAT array is proposed. For electromagnetic simulation, analytical solutions to a meander coil are proposed and verified. By comparing with FEM, the analytical method provides several advantages, especially at high frequencies. The calculated Lorentz force density is used as the excitation source in the ultrasonic simulation. The results show that the maximum energy of surface waves is concentrated at the steering angle $0^{\circ}$ and $180^{\circ}$, and occurs at the positions where the EMAT sensor is located.

\section{Acknowledgement}

This work was financially supported by Engineering and Physical Sciences Research Council (Grant No. EP/M020835/1). We thank our colleagues from the School of Electrical and 
Electronic Engineering in University of Manchester. All data created during this research are openly available from Zenodo at http://dx.doi.org/10.5281/zenodo.54981.

\section{References}

1. Cacciola, M., et al., Fuzzy characterization of flawed metallic plates with eddy current tests. Progress In Electromagnetics Research, 2007. 72: p. 241-252.

2. Lovejoy, D., Magnetic Particle Inspection: A Practical Guide. David Lovejoy. 1993: Springer.

3. Mahmoudi, M. and S.Y. Tan, Depth detection of conducting marine mines via eddy-current and current-channeling response. Progress In Electromagnetics Research, 2009. 90: p. 287-307.

4. Park, M.H., I.S. Kim, and Y.K. Yoon, Ultrasonic inspection of long steel pipes using Lamb waves. NDT \& E International, 1996. 29(1): p. 13-20.

5. Yin, W. and A. Peyton, Thickness measurement of non-magnetic plates using multi-frequency eddy current sensors. NDT \& E International, 2007. 40(1): p. 43-48.

6. Yin, W. and A.J. Peyton, Thickness measurement of metallic plates with an electromagnetic sensor using phase signature analysis. Instrumentation and Measurement, IEEE Transactions on, 2008. 57(8): p. 1803-1807.

7. Zhao, Q., J. Hao, and W. Yin, A simulation study of flaw detection for rail sections based on high frequency magnetic induction sensing using the boundary element method. Progress In Electromagnetics Research, 2013. 141: p. 309-325.

8. Cawley, P. Ultrasonic measurements for the quantitative NDE of adhesive joints-potential and challenges. in Ultrasonics Symposium, 1992. Proceedings., IEEE 1992. 1992. IEEE.

9. Chassignole, B., et al. Characterization of austenitic stainless steel welds for ultrasonic NDT. in REVIEW OF PROGRESS IN QUANTITATIVE NONDESTRUCTIVE EVALUATION: Volume 19. 2000. AIP Publishing.

10. Drinkwater, B. and P. Cawley, Measurement of the frequency dependence of the ultrasonic reflection coefficient from thin interface layers and partially contacting interfaces. Ultrasonics, 1997. 35(7): p. 479-488.

11. Drinkwater, B.W. and P.D. Wilcox, Ultrasonic arrays for non-destructive evaluation: A review. NDT \& E International, 2006. 39(7): p. 525-541.

12. Gallego-Juarez, J., Piezoelectric ceramics and ultrasonic transducers. Journal of Physics E: Scientific Instruments, 1989. 22(10): p. 804.

13. Jaffe, H. and D. Berlincourt, Piezoelectric transducer materials. Proceedings of the IEEE, 1965. 53(10): p. 1372-1386.

14. Jaffe, B., Piezoelectric ceramics. Vol. 3. 2012: Elsevier.

15. Caratelli, D., A. Lay-Ekuakille, and P. Vergallo, Non-Invasive Reflectometry-Based Detection of Melanoma by Piezoelectric Micro-Needle Antenna Sensors. Progress In Electromagnetics Research, 2013. 135: p. 91-103.

16. Ribichini, R., MODELLING OF ELECTROMAGNETIC ACOUSTIC TRANSDUCERS (Ph.D. Dissertation), 2011, Imperial College London.

17. Dhayalan, R. and K. Balasubramaniam, A hybrid finite element model for simulation of electromagnetic acoustic transducer (EMAT) based plate waves. NDT \& E International, 2010. 43(6): p. 519-526.

18. Dixon, S., C. Edwards, and S. Palmer, High accuracy non-contact ultrasonic thickness gauging of aluminium sheet using electromagnetic acoustic transducers. Ultrasonics, 2001. 39(6): p. 445-453.

19. Nakamoto, H., et al., Reliability evaluation of pipe thickness measurement by electromagnetic acoustic transducer. International Journal of Applied Electromagnetics and Mechanics, 2014. 45(1-4): p. 923-929. 
20. Tone, R., Y. Tanaka, and Y. Fujimoto, Experiment of receiving ultrasonic wave by using adhesion and a contact type PVDF sensor. International Journal of Applied Electromagnetics and Mechanics, 2014. 45(1-4): p. 171-177.

21. Wu, X., X. Ding, and L. Wang, An improved longitudinal wave EMAT based on the shielding effect. International Journal of Applied Electromagnetics and Mechanics, 2014. 45(1-4): p. 227-233.

22. Wang, S., et al., 3-D modeling and analysis of meander-line-coil surface wave EMATs. Mechatronics, 2012. 22(6): p. 653-660.

23. Hirao, M. and H. Ogi, EMATs for science and industry: noncontacting ultrasonic measurements. 2003: Springer Science \& Business Media.

24. Luo, W. and J. Rose, Guided wave thickness measurement with EMATs. Insight-NonDestructive Testing and Condition Monitoring, 2003. 45(11): p. 735-739.

25. Dhayalan, R. and K. Balasubramaniam, A two-stage finite element model of a meander coil electromagnetic acoustic transducer transmitter. Nondestructive Testing and Evaluation, 2011. 26(02): p. 101-118.

26. Scruby, C. and B. Moss, Non-contact ultrasonic measurements on steel at elevated temperatures. NDT \& E International, 1993. 26(4): p. 177-188.

27. Edwards, R., S. Dixon, and X. Jian. Non - Contact Ultrasonic Characterization of Defects Using EMATS. in REVIEW OF PROGRESS IN QUANTITATIVE NONDESTRUCTIVE EVALUATION. 2005. American Institute of Physics.

28. Latimer, P.J. and D.T. MacLauchlan, EMAT probe and technique for weld inspection, 1998, Google Patents.

29. Ludwig, R., Z. You, and R. Palanisamy, Numerical simulations of an electromagnetic acoustic transducer-receiver system for NDT applications. Magnetics, IEEE Transactions on, 1993. 29(3): p. 2081-2089.

30. Thomas, S., et al., A coupled electromagnetic and mechanical analysis of electromagnetic acoustic transducers. International Journal for Computational Methods in Engineering Science and Mechanics, 2009. 10(2): p. 124-133.

31. Kaltenbacher, M., et al., Finite element analysis of coupled electromagnetic acoustic systems. Magnetics, IEEE Transactions on, 1999. 35(3): p. 1610-1613.

32. Murayama, R. and K. Mizutani, Conventional electromagnetic acoustic transducer development for optimum Lamb wave modes. Ultrasonics, 2002. 40(1): p. 491-495.

33. Jian, X., et al., A model for pulsed Rayleigh wave and optimal EMAT design. Sensors and Actuators A: Physical, 2006. 128(2): p. 296-304.

34. Dhayalan, R., et al., A hybrid finite element model for spiral coil electromagnetic acoustic transducer (EMAT). International Journal of Applied Electromagnetics and Mechanics, 2014. 46(3): p. 491-500.

35. Dodd, C. and W. Deeds, Analytical Solutions to Eddy - Current Probe - Coil Problems. Journal of applied physics, 1968. 39(6): p. 2829-2838.

36. Garcia-Rodriguez, D., O. Mihalache, and M. Ueda, EMAT simulations based on a twodimensional FEM coupled electro-mechanical formulation. International Journal of Applied Electromagnetics and Mechanics, 2014. 45(1): p. 543-549.

37. Kang, L., et al., Enhancement of signal amplitude of surface wave EMATs based on 3-D simulation analysis and orthogonal test method. NDT \& E International, 2013. 59: p. 11-17.

38. Lu, C. and Z.-d. Zhang. Finite-difference simulation of rayleigh wave scattering at a surface crack with experimental verification. in Piezoelectricity, Acoustic Waves, and Device Applications, 2008. SPAWDA 2008. Symposium on. 2008. IEEE.

39. Wang, S., et al., A novel method for modeling and analysis of meander-line-coil surface wave EMATs, in Life System Modeling and Intelligent Computing. 2010, Springer. p. 467-474. 
40. Wang, S., et al., Influence of Coil Parameters on Rayleigh Waves Excited by Meander-Line Coil EMATs, in Intelligent Computing for Sustainable Energy and Environment. 2013, Springer. p. 94-103.

41. Xie, Y., et al., Simulation of ultrasonic and EMAT arrays using FEM and FDTD. Ultrasonics, 2016. 66: p. 154-165.

42. Xie, Y., et al., Simulation of an Electromagnetic Acoustic Transducer Array by using Analytical method and FDTD. Journal of Sensors, 2016. 501: p. 5451821.

43. Nakano, H., Some problems concerning the propagation of the disturbances in and on semiinfinite elastic solid. Geophys. Mag, 1930. 2: p. 189-348. 
Table 1. Summary of method used for modelling EMAT.

\begin{tabular}{|c|c|c|c|c|c|}
\hline \multirow{2}{*}{ People } & \multicolumn{2}{|c|}{ Electromagnetic simulation } & \multicolumn{3}{|c|}{ Ultrasonic simulation } \\
\hline & FEM & Analytical method & FEM & FDTD & Analytical method \\
\hline $\begin{array}{c}{[33,37,39} \\
40]\end{array}$ & O & & & & 0 \\
\hline$[17,25]$ & 0 & & 0 & & \\
\hline Authors[41] & 0 & & & 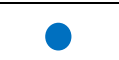 & \\
\hline Authors[42] & & O & & O & \\
\hline Authors & & 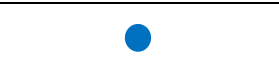 & & & 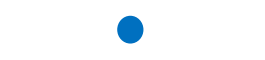 \\
\hline
\end{tabular}


Table 2. Parameters used for studying the analytical solutions.

\begin{tabular}{|c|c|c|c|c|c|}
\hline Description & Symbol & Value & Description & Symbol & Value \\
\hline The length of the aluminium & $R$ & $10 \mathrm{~mm}$ & Lift-off & $l$ & $1 \mathrm{~mm}$ \\
\hline The height of the aluminium & $H$ & $10 \mathrm{~mm}$ & $\begin{array}{c}\text { Applied current } \\
\text { density }\end{array}$ & $\mathrm{I}$ & $1 \mathrm{~A} / \mathrm{m}^{2}$ \\
\hline $\begin{array}{l}\text { Inside radius of the circular } \\
\text { coil }\end{array}$ & $r_{1}$ & $4.95 \mathrm{~mm}$ & $\begin{array}{l}\text { Outside radius of } \\
\text { the circular coil }\end{array}$ & $r_{2}$ & $5.05 \mathrm{~mm}$ \\
\hline Mean radius of the spiral coil & $\frac{r_{1}+r_{2}}{2}$ & $5 \mathrm{~mm}$ & Frequency & $\mathrm{f}$ & $1 \mathrm{kHz}$ \\
\hline The height of the coil & $h$ & $1 \mathrm{~mm}$ & $\begin{array}{l}\text { Conductivity of } \\
\text { aluminium }\end{array}$ & $\sigma$ & $\begin{array}{c}38000000 \\
\text { siemens } / \mathrm{m}\end{array}$ \\
\hline Permeability of air & $\mu_{0}$ & $\begin{array}{c}1.257 \times 10^{-6} \\
\mathrm{H} / \mathrm{m}\end{array}$ & $\begin{array}{l}\text { Permeability of } \\
\text { aluminium }\end{array}$ & $\mu_{1}$ & $\begin{array}{c}1.257 \times 10^{-6} \\
\mathrm{H} / \mathrm{m}\end{array}$ \\
\hline
\end{tabular}


Figure 1. The geometry used for eddy current calculation.

Figure 2. The magnitude distribution of the vector potential A under a circular coil.

Figure 3. The magnitude distribution of the vector potential A under a straight wire.

Figure 4. The magnitude distribution of the vector potential based on the numerical method (a) and the analytical method (b).

Figure 5. With an operating frequency of $1 \mathrm{kHz}$, the distribution of the vector potential along the surface of aluminium; (a), the magnitude distribution, (b), the real part distribution, (c), the imaginary distribution.

Figure 6. With an operating frequency of $1 \mathrm{MHz}$, the distribution of the vector potential along the surface of aluminium; (a), the magnitude distribution, (b), the real part distribution, (c), the imaginary part distribution.

Figure 7. With an operating frequency $1 \mathrm{kHz}$, the distribution of the vector potential at a depth of 3mm; (a), the magnitude distribution, (b), the real part distribution, (c), the imaginary distribution.

Figure 8. The configuration of a typical EMAT.

Figure 9. The distribution of the vector potential based on the analytical method.

Figure 10. Distribution of Lorentz force density along the surface of the stainless steel plate.

Figure 11. The displacement of surface waves caused by a surface point force.

Figure 12. Transformation from electromagnetic model to ultrasonic model.

Figure 13. Surface waves distribution on $x-y$ section. (a), the amplitude distribution of surface waves; (b), the geometry used for studying beam features.

Figure 14. Beam features of surface waves based on the surface waves' distribution. 


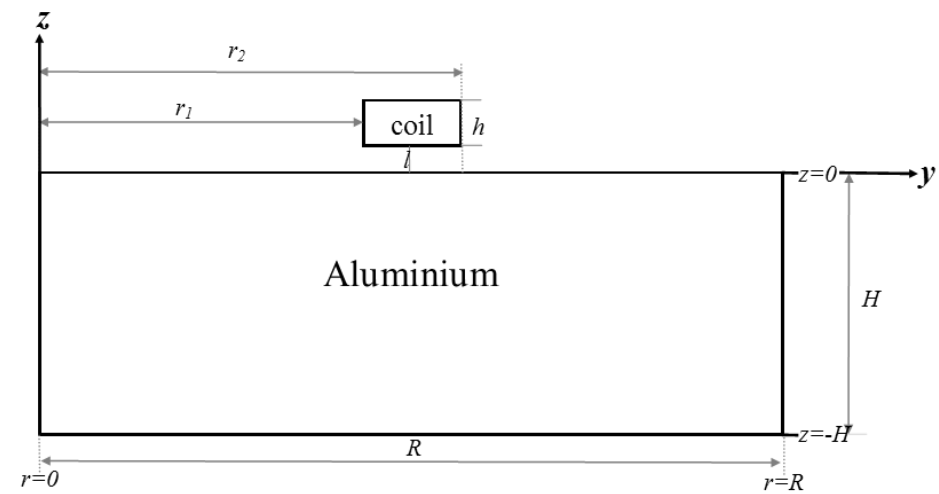

Figure 1. The geometry used for eddy current calculation. 


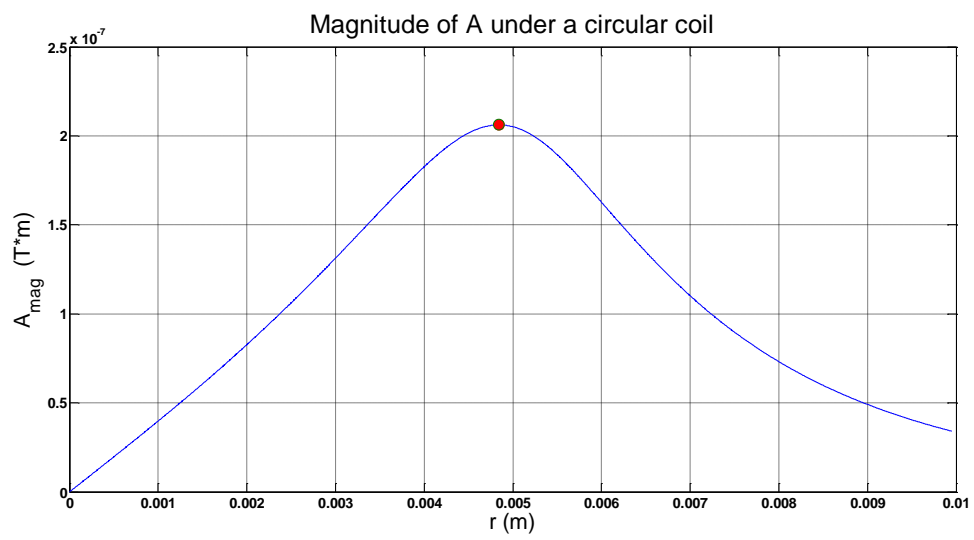

Figure 2. The magnitude distribution of the vector potential A under a circular coil. 


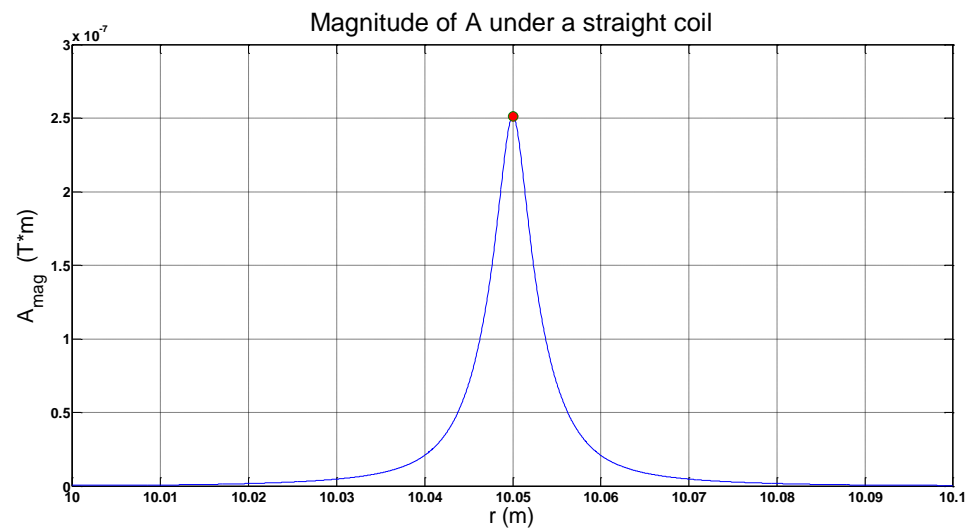

Figure 3. The magnitude distribution of the vector potential A under a straight wire. 
(a)

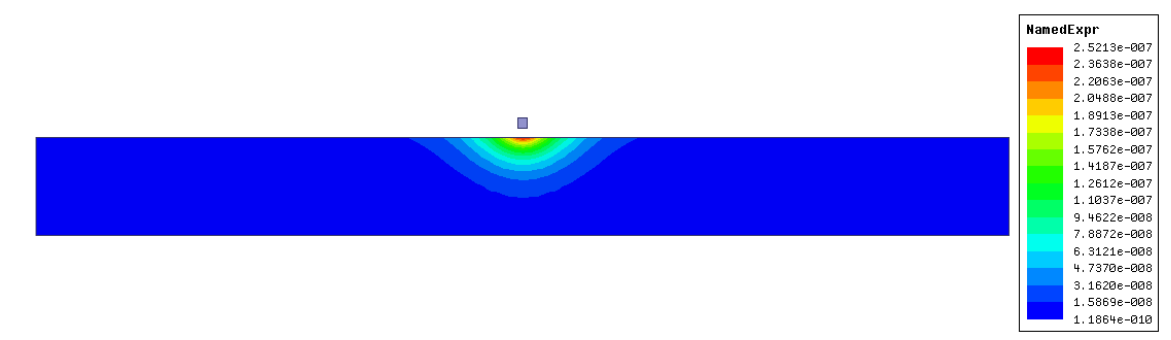

(b)

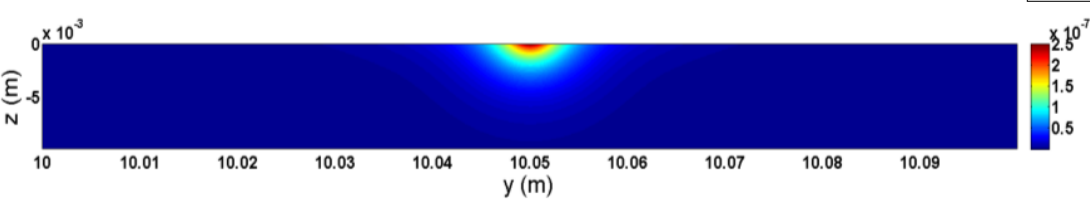

Figure 4. The magnitude distribution of the vector potential based on the numerical method (a) and the analytical method (b). 

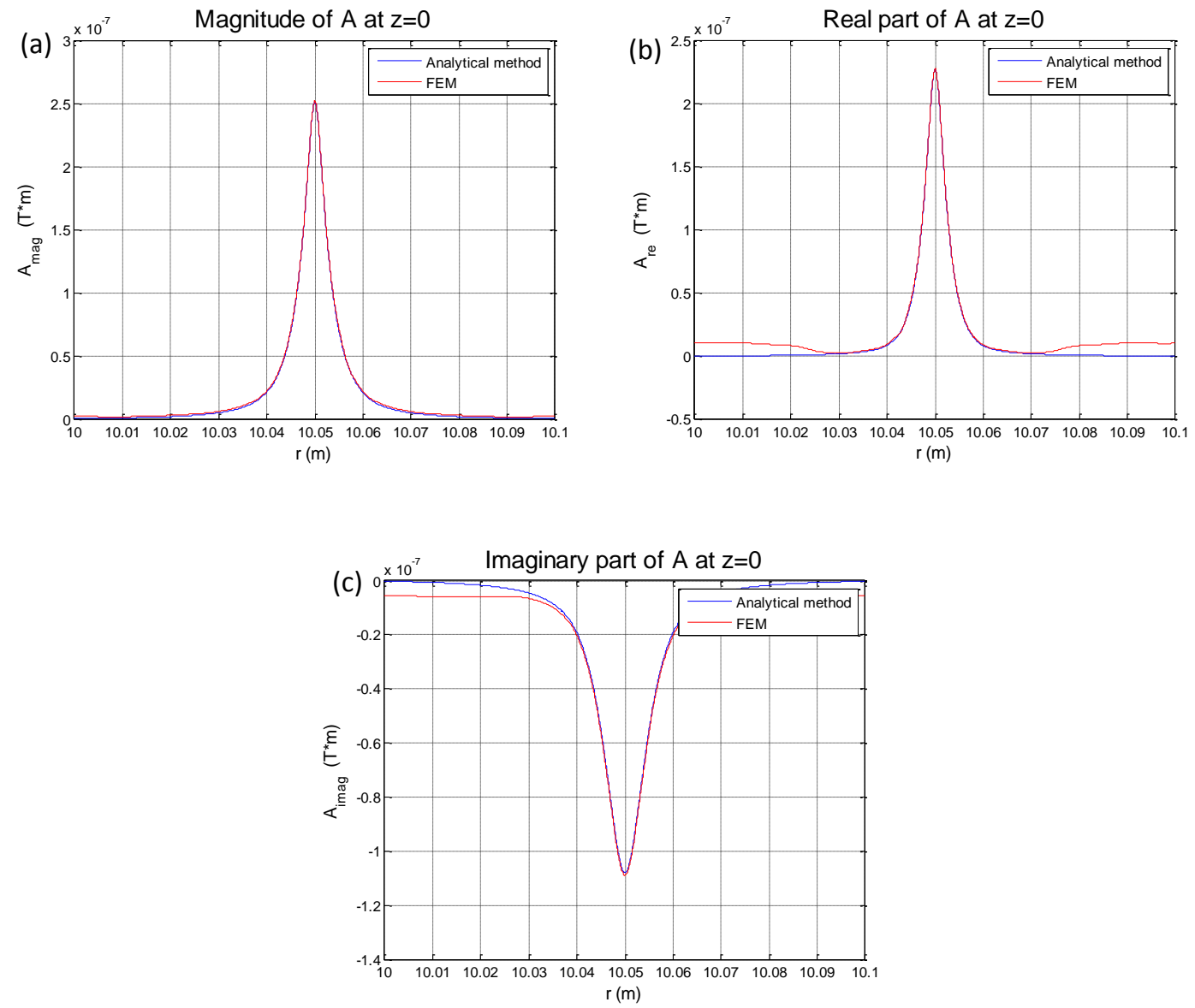

Figure 5. With an operating frequency of $1 \mathrm{kHz}$, the distribution of the vector potential along the surface of aluminium; (a), the magnitude distribution, (b), the real part distribution, (c), the imaginary distribution. 

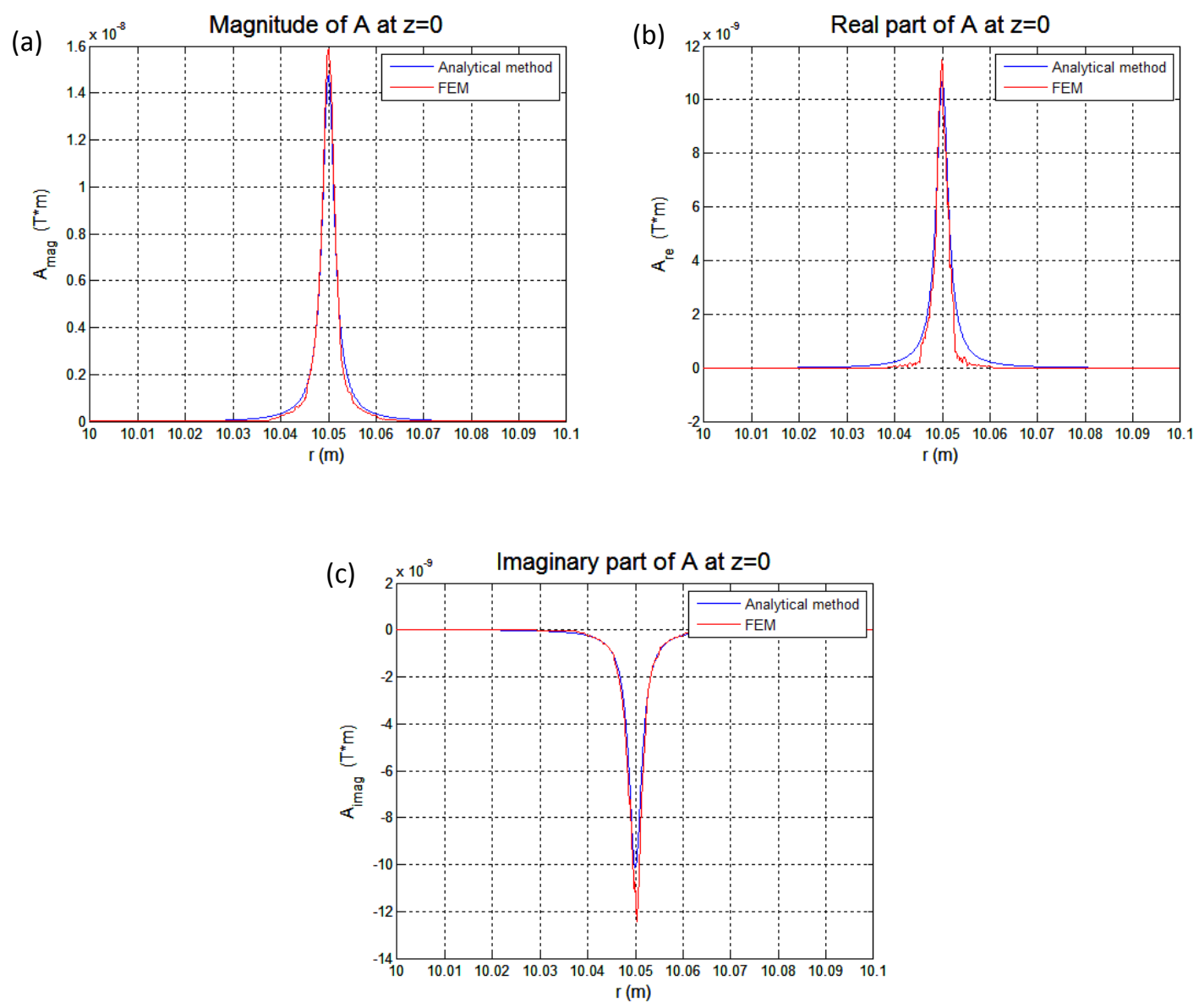

Figure 6. With an operating frequency of $1 \mathrm{MHz}$, the distribution of the vector potential along the surface of aluminium; (a), the magnitude distribution, (b), the real part distribution, (c), the imaginary part distribution. 

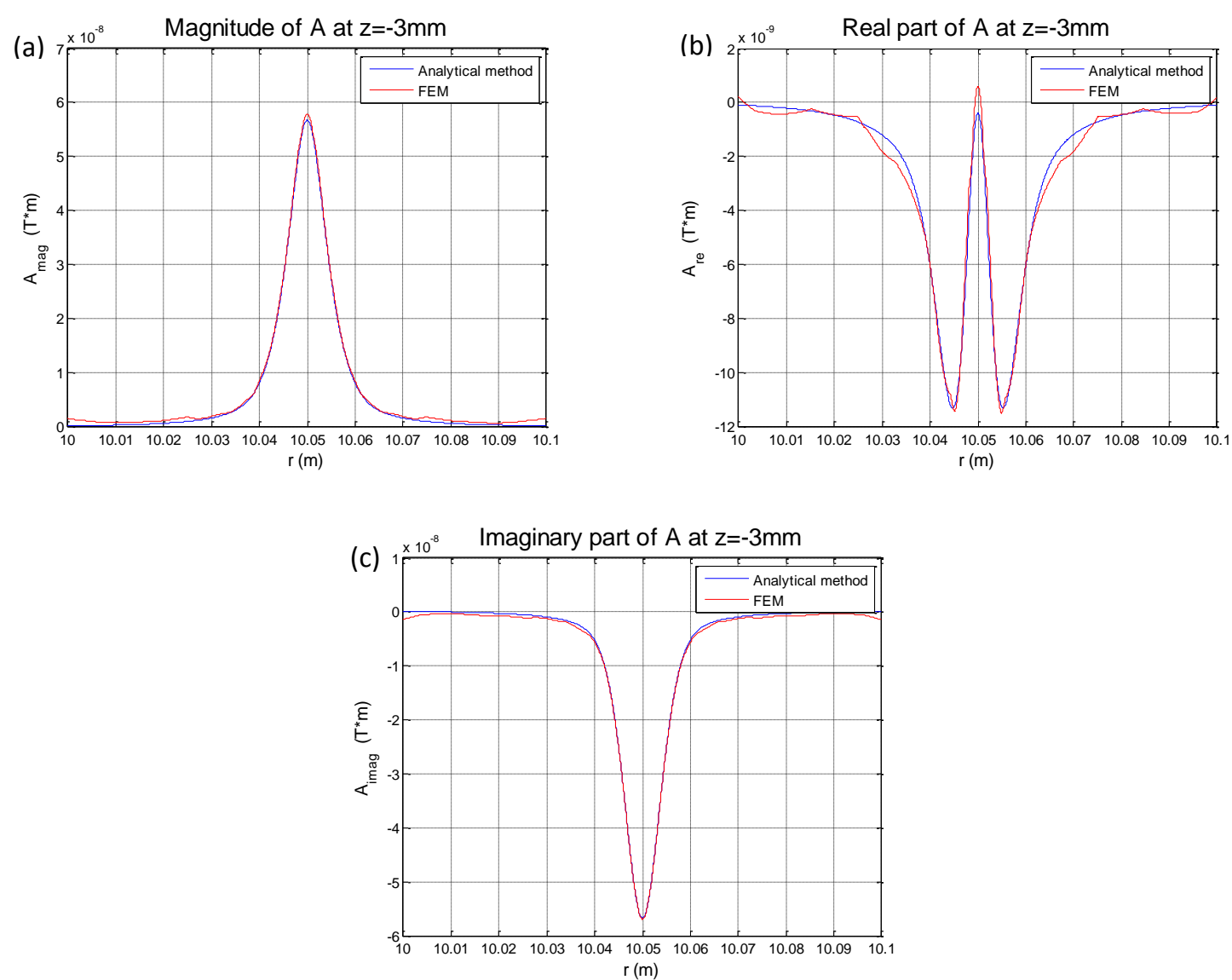

Figure 7. With an operating frequency $1 \mathrm{kHz}$, the distribution of the vector potential at a depth of 3mm; (a), the magnitude distribution, (b), the real part distribution, (c), the imaginary distribution. 


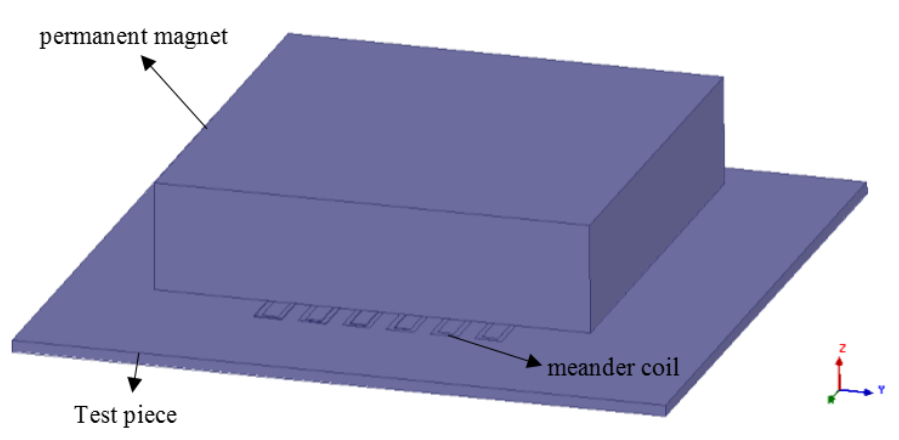

Figure 8. The configuration of a typical EMAT. 


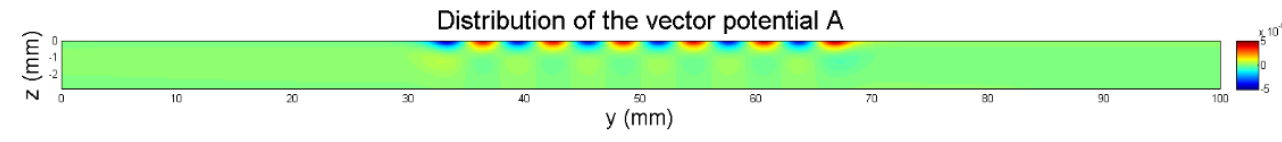

Figure 9. The distribution of the vector potential based on the analytical method. 


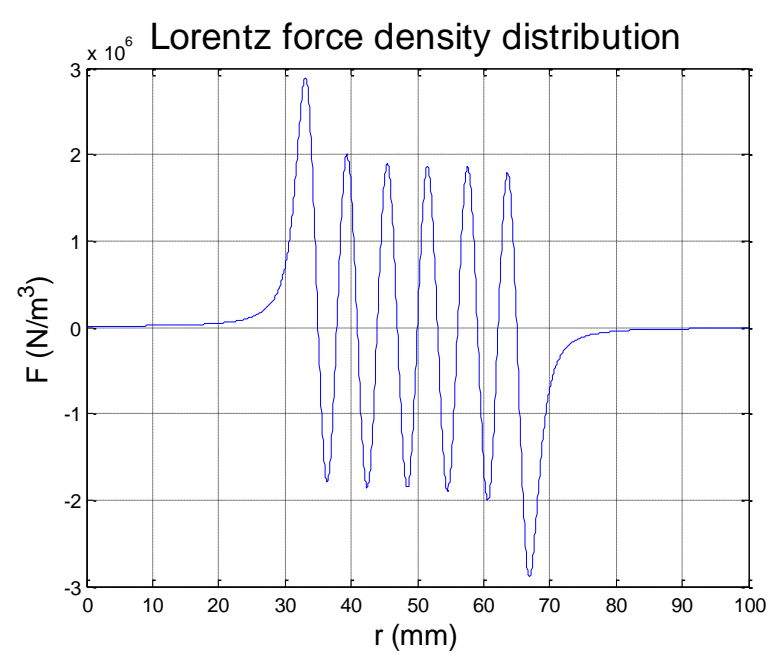

Figure 10. Distribution of Lorentz force density along the surface of the stainless steel plate. 


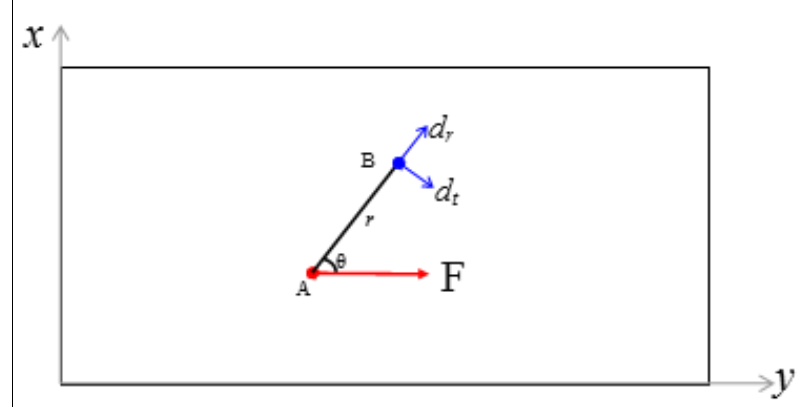

Figure 11. The displacement of surface waves caused by a surface point force. 
Electromagnetic model

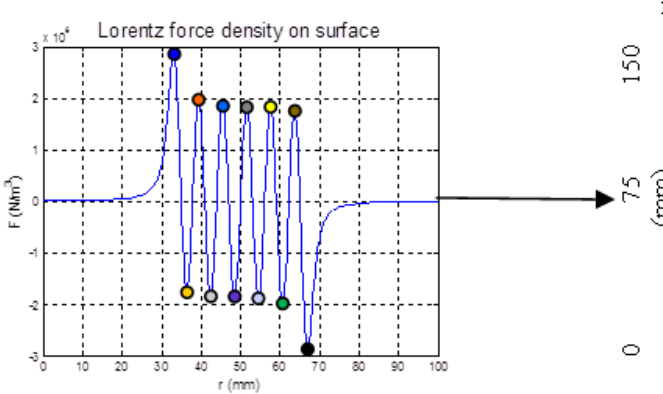

Ultrasonic model

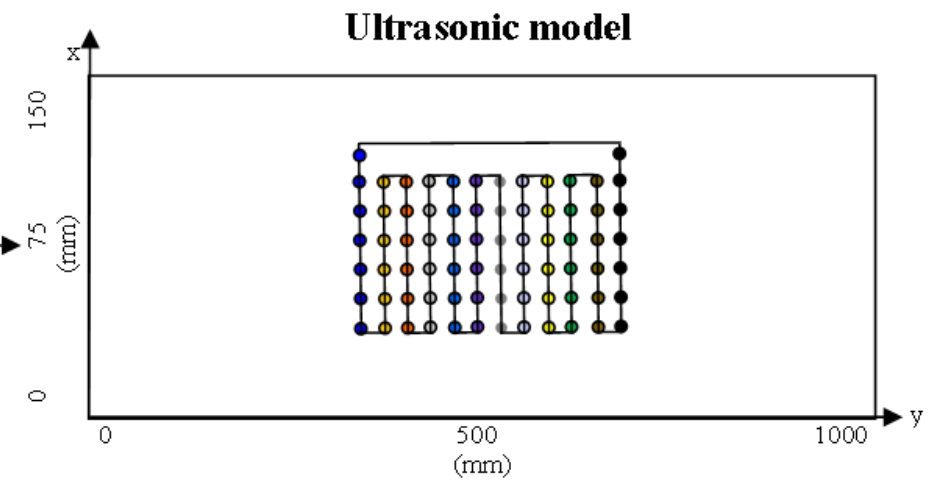

Figure 12. Transformation from electromagnetic model to ultrasonic model. 
(a)

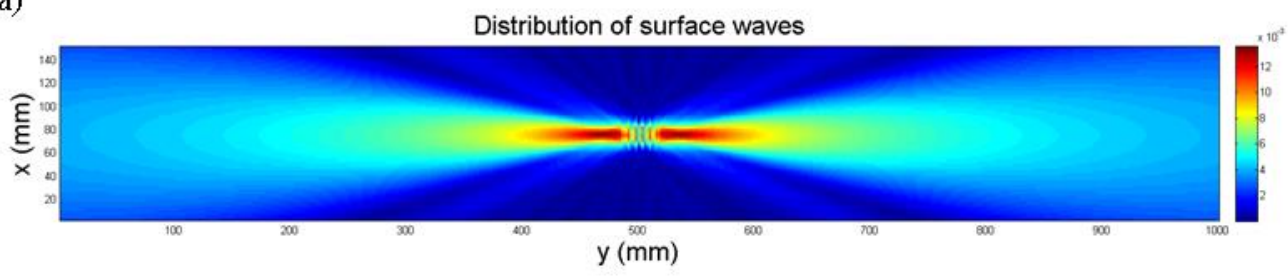

(b)

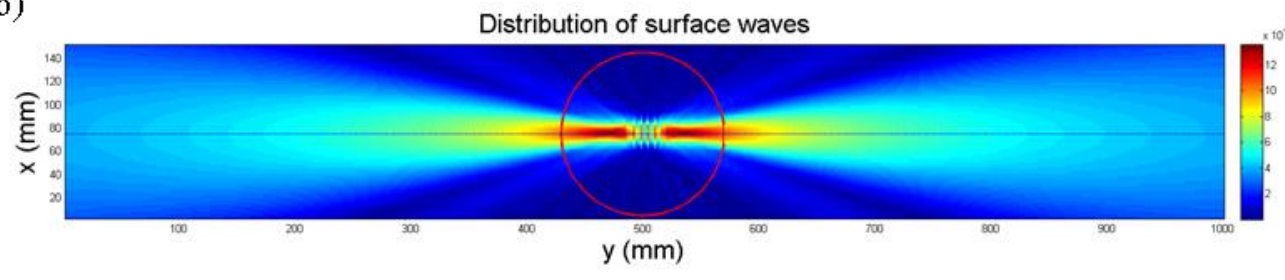

Figure 13. Surface waves distribution on $x-y$ section. (a), the amplitude distribution of surface waves; (b), the geometry used for studying beam features. 
(a)

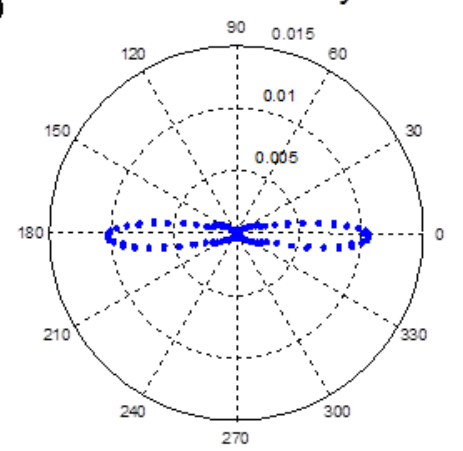

(b)

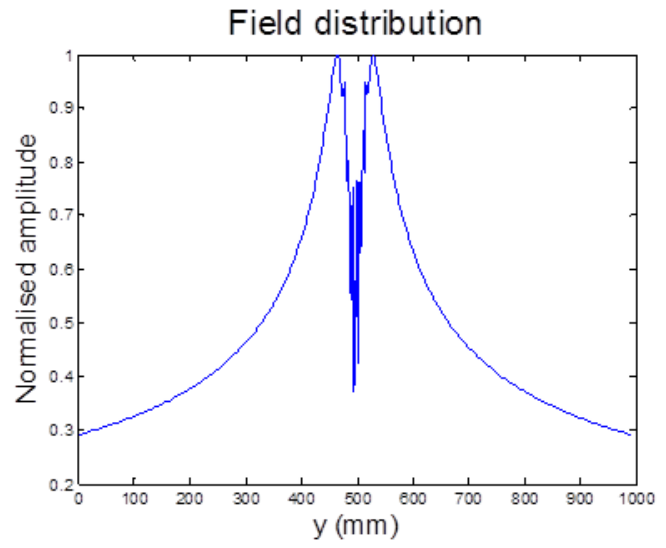

Figure 14. Beam features of surface waves based on the surface waves' distribution. 